\title{
Perforated Gastrointestinal Stromal Tumor of the Small Intestine: A Case Report Presenting With Peritonitis
}

\author{
Daniel Paramythiotis ${ }^{\mathrm{a}}$, Stavros Panidis ${ }^{\mathrm{a}, \mathrm{c}}$, Vasilis Papadopoulos ${ }^{\mathrm{a}}$, Dimitris Panagiotou, \\ Pavlina Panteliadou ${ }^{\mathrm{b}}$, Antonios Michalopoulos ${ }^{\mathrm{a}}$
}

\begin{abstract}
A case of acute abdomen caused by perforated gastrointestinal stromal tumor (GIST) of small intestine is described. A 56 years old man presented to the emergency room with acute abdominal pain. An abdominal CT showed collections of free air in the abdomen with obstruction of the distal small intestine. Laparotomy revealed perforation of a tumor in the jejunum. Pathologic examination of the lesion showed that the tumor was GIST. Postoperatively, the patient received treatment using imatinib. GISTs are rare and they don't have any specific symptoms. Most common indications for surgery include bleeding and obstruction, although free perforation may occur as a result of hemorrhagic necrosis in large tumor masses. Complete surgical resection is the primary treatment option, followed by adjuvant therapy with imatinib if necessary.
\end{abstract}

Keywords: Perforation; GISTs; Small intestine; Imatinib

\section{Introduction}

The most common malignant neoplasms of the small bowel in the approximate order of frequency are adenocarcinomas, carcinoid tumors, malignant gastrointestinal stromal tumors

Manuscript accepted for publication August 11, 2011

\footnotetext{
${ }^{a} 1$ st Propedeutic Department of Surgery, AHEPA University Hospital, Aristotle University of Thessaloniki, St. Kyriakidi 1 54636, Thessaloniki, Greece

${ }^{\mathrm{b}}$ Department of Pathology, Aristotle University of Thessaloniki, St. Kyriakidi 1 54636, Medical School, Thessaloniki, Greece

${ }^{\mathrm{c} C}$ Corresponding author: Stavros Panidis, 28th oktober 17 street, 56625

Thessaloniki, Greece. Email: st.panidis@gmail.com
}

doi:10.4021/jcs102e
(GISTs) and lymphomas.

Malignant GISTs which arise from mesenchymal tissue, constitute about $20 \%$ of malignant neoplasms of the small bowel. It is estimated that the frequency of these tumors is $10-20 / 1,000,000$ population $[1,2]$ and the possibility of presence of malignancy is $20-30 \%$. Malignant GISTs are greater than $5 \mathrm{~cm}$ at the time of diagnosis in $80 \%$ of patients. GISTs mostly arise from the muscolaris propria and generally grow extramurally. Most common indications for surgery include bleeding and obstruction, although free perforation may occur as a result of hemorrhagic necrosis in large tumor masses. Typically, GISTs tend to invade locally and spread by direct extension into adjacent tissues and hematogenously to the liver, lungs, and bone; lymphatic metastases are unusual. The most useful indicators of survival and the risk for metastasis include the size of the tumor at presentation, the mitotic index, and evidence of tumor invasion into the lamina propria.

\section{Case Report}

A 56 year old man presented with diffuse abdominal pain and abdominal distention with associated nausea. There were no other significant signs and symptoms. He was admitted with diagnosis of acute abdomen. Routine blood

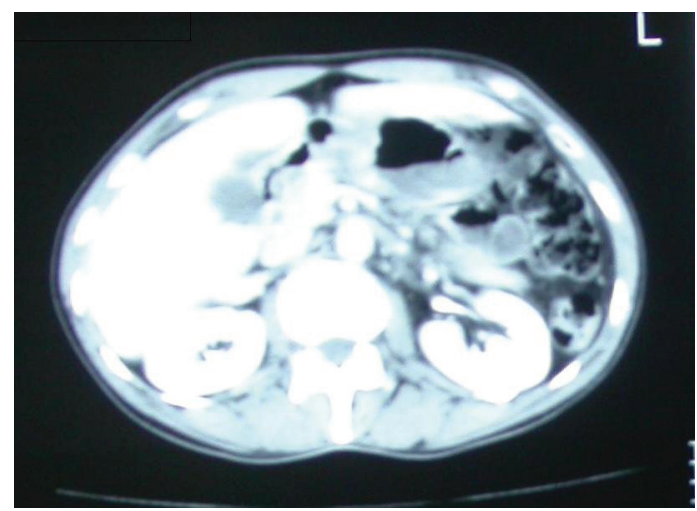

Figure 1. Abdominal ct revealing, free air in the abdominal cavity. 


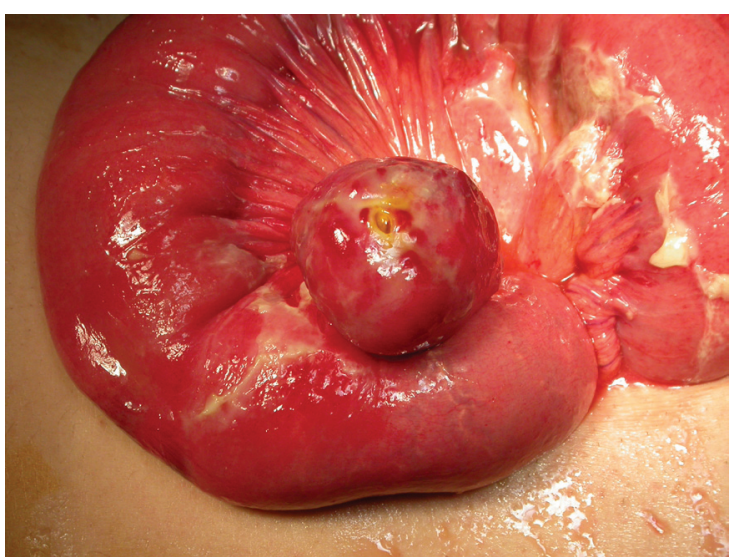

Figure 2. Intraoperative photo showing the tumor and the site of perforation.

tests were normal and showed only elevated white blood cell count (WBC). The patient had a personal history of chronic anemia. He subsequently underwent abdominal ultrasound and CT imaging that showed dilated loops of the small intestine with transitions to nondilated loops, suggesting an obstruction in the mid to distal small bowel. A small amount of liquid and several small collections of apparent free air were evident within the abdomen (Fig. 1).

The laparotomy revealed large amount of free air and purulent fluid in the peritoneal cavity. The distal part of the jejunum showed perforation of a small intestinal tumor of maximum diameter $3 \mathrm{~cm}$ (Fig. 2). The tumor and $13 \mathrm{~cm}$ of the ileum were excised and enteroenteroanastomosis were performed. The inspection of the rest gastrointestinal tract and peritoneal cavity did not reveal other abnormalities. The patient had a postoperative course without complications and was discharged from the hospital on the 7th post-operative day. Cytopathological examination revealed a gastrointestinal stromal tumor (GIST) (Fig. 3). The tumor cells were spindleshaped and arranged in ill-defined fascicles, sometimes in a storiform pattern. They had an oval nucleus, centrally placed and prominent cytoplasmic vacuole. Nuclear atypia and tumor necrosis were not observed. Mitoses were $<5$ per 50 high power fields. Immunohistochemically, the tumor cells were positive for CD117/c-kit and CD34. Few tumor cells were also positive for smooth muscle actin and S-100 protein. Postoperatively the patient was treated by oral administration of $400 \mathrm{mg}$ imatinib once a day. He is free of any signs of recurrence 48 months after the operation.

\section{Discussion}

GISTs predominantly occur in patients around the sixth decade of life and can be found in any site of gastrointestinal track with no significant difference in distribution between males and females [2].

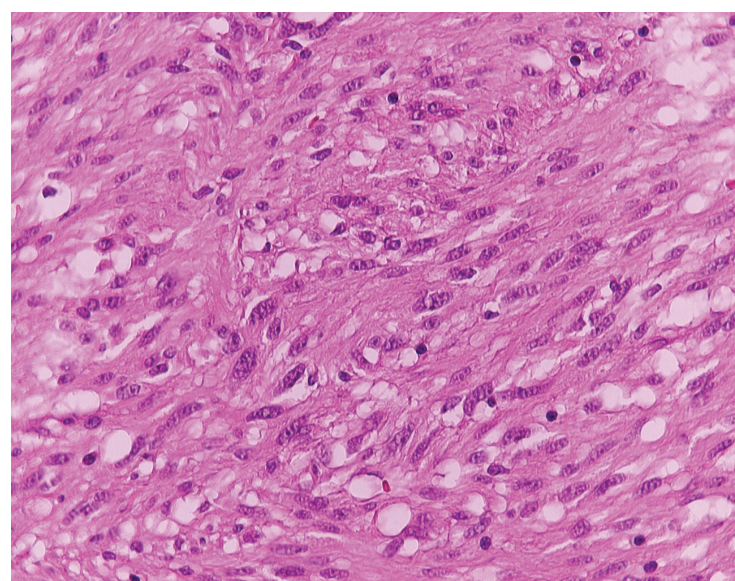

Figure 3. Pathological slide.

The symptoms, which depend on tumor size and location, are usually non specific [3]. Small GISTs are usually asymptomatic and are detected either during investigations or surgical procedures for unrelated disease. Incidental discovery accounts for approximately one third of the cases. The most common presentation is bleeding related to mucosal erosion (approximately 50\%) [3], which may be either chronic, with anemia, or acute, necessitating emergency treatment (approximately $40 \%$ of the cases presenting with hemorrhage). Some $20 \%$ of patients present abdominal discomfort, or even pain, which is generally associated with larger size GIST. In the small intestine obstruction is also frequent. Bowel perforation is infrequent. The symptoms and signs are not disease specific and as a consequence about $50 \%$ of GISTs have already metastases at the time of diagnosis, usually to the liver or the peritoneum $[1,4,5]$.

Although the diagnostic procedure includes several examinations, such as barium examination of the gastrointestinal track, CT and angiography, none of these can establish the correct diagnosis with $100 \%$ certainty. The preoperative percutaneous fine needle aspiration of the tumor for diagnostic purpose is not indicated because of the danger of potential intraperitoneal migration of tumor cells $[1,6,7]$. Recently, several studies pointed out the significance of endoscopic ultrasound-guided fine-needle aspiration for the diagnosis of GIST with reported accuracy of $89 \%$. On the other hand, positron emission tomography (PET) with 18 Fluoro2D glucose is a very useful tool for the postoperative follow-up of patients receiving imatinib.

The diagnosis of GIST may be suggested during surgery by the presence of a well defined extraluminal mass, frequently polylobulated with a pseudocapsule. However, it always requires histological and immunohistochemical confirmation.

GISTs can be categorized as low or high risk tumors based on a scale which is composed of five criteria (tumor size $>5 \mathrm{~cm}$, mitotic rate $>5 / 50 \mathrm{HPF}$, Mib1 $>10 \%$, presence 
of necrosis and invasive components to mucosa or serosa), presence of either lymph node invasion or metastases [8]. With this scale, the 5-year survival of low malignant potential GIST is $95 \%$ and of high malignant potential Gist less than $20 \%$.

The first step in the treatment of GISTs is the surgical $\mathrm{R} 0$ resection of the primary tumor. Several reviews have reported that small GISTs $(<2 \mathrm{~cm})$ can be treated adequately by wedge (gastric) or segmental (bowel) resection. Larger GISTs may require more extensive resection including adjacent structures or organs if involved [10]. Surgical resection is associated with a 5-year survival rate of $48-70 \%$. There is no indication for chemotherapy and radiation therapy after surgical resection of GISTs as these tumors are notoriously unresponsive to such treatment. Radiotherapy is only used in cases of intraperitoneal hemorrhage, when the precise location of the tumor is known or for analgesic purposes [1, 11]. In advanced tumors, a neoadjuvant strategy to reduce the tumor load before surgical resection is under study. The molecular status of GISTs turns out to be relevant for the response to targeted treatment with imatinib a powerful and relatively selective inhibitor of all $\mathrm{ABL}$ tyrosine kinases of platelet-derived growth factor receptor (PDGFR) and of c-kit receptor. Imatinib has also become the first line of treatment for recurrent and/or metastatic GISTs and its very well tolerated (Oral administration of $400 \mathrm{mg}$ daily for some months). Longer duration of imatinib treatment in patients with advanced GIST increase the risk of resistance to therapy. Several groups describe an increasing frequency of secondary mutations in KIT or PDGFRa. Several other mechanisms as for example, gene amplification or activation of other tyrosine kinase pathways are still under research $[12,14]$.

\section{Conclusions}

Surgical resection without extensive lymph node sampling is the treatment of choice in the treatment of GISTs. But even this has resulted in poor outcome and recurrence in patients with malignant potential GISTs or with complications such as perforated tumors, metastatic and unresectable tumors and locoregional recurrence. In advanced cases the gold standard is a targeted therapy with tyrosine kinase inhibitors leading to response rates of up to $80 \%$.

\section{References}

1. Connolly EM, Gaffney E, Reynolds JV. Gastrointestinal stromal tumours. Br J Surg. 2003;90(10):1178-1186.
2. Miettinen M, Majidi M, Lasota J. Pathology and diagnostic criteria of gastrointestinal stromal tumors (GISTs): a review. Eur J Cancer. 2002;38 Suppl 5:S3951.

3. Ludwig DJ, Traverso LW. Gut stromal tumors and their clinical behavior. Am J Surg. 1997;173(5):390-394.

4. DeMatteo RP, Lewis JJ, Leung D, Mudan SS, Woodruff JM, Brennan MF. Two hundred gastrointestinal stromal tumors: recurrence patterns and prognostic factors for survival. Ann Surg. 2000;231(1):51-58.

5. Emory TS, Sobin LH, Lukes L, Lee DH, O'Leary TJ. Prognosis of gastrointestinal smooth-muscle (stromal) tumors: dependence on anatomic site. Am J Surg Pathol. 1999;23(1):82-87.

6. Efremidou EI, Liratzopoulos N, Papageorgiou MS, Romanidis K. Perforated GIST of the small intestine as a rare cause of acute abdomen: Surgical treatment and adjuvant therapy. Case report. J Gastrointestin Liver Dis. 2006;15(3):297-299.

7. Bucher P, Villiger P, Egger JF, Buhler LH, Morel P. Management of gastrointestinal stromal tumors: from diagnosis to treatment. Swiss Med Wkly. 2004;134(1112):145-153.

8. Yan H, Marchettini P, Acherman YI, Gething SA, Brun E, Sugarbaker PH. Prognostic assessment of gastrointestinal stromal tumor. Am J Clin Oncol. 2003;26(3):221228.

9. Miettinen M, Lasota J. Gastrointestinal stromal tumors: review on morphology, molecular pathology, prognosis, and differential diagnosis. Arch Pathol Lab Med. 2006;130(10):1466-1478.

10. Roberts PJ, Eisenberg B. Clinical presentation of gastrointestinal stromal tumors and treatment of operable disease. Eur J Cancer. 2002;38 Suppl 5:S37-38.

11. Aparicio T, Boige V, Sabourin JC, Crenn P, Ducreux M, Le Cesne A, Bonvalot S. Prognostic factors after surgery of primary resectable gastrointestinal stromal tumours. Eur J Surg Oncol. 2004;30(10):1098-1103.

12. Wardelmann E, Buttner R, Merkelbach-Bruse S, Schildhaus HU. Mutation analysis of gastrointestinal stromal tumors: increasing significance for risk assessment and effective targeted therapy. Virchows Arch. 2007;451(4):743-749.

13. Debiec-Rychter M, Cools J, Dumez H, Sciot R, Stul M, Mentens N, Vranckx H, et al. Mechanisms of resistance to imatinib mesylate in gastrointestinal stromal tumors and activity of the PKC412 inhibitor against imatinibresistant mutants. Gastroenterology. 2005;128(2):270279. 